\title{
Biología y pesquería del pulpo Octopus vulgaris (Octopoda: Octopodidae) en las costas del estado Nueva Esparta, Venezuela
}

\author{
Leo Walter González ${ }^{1}$, Nora Eslava ${ }^{1}$, Francisco Guevara $^{1} \&$ Luís Troccoli ${ }^{2}$ \\ 1. Área de Biología y Recursos Pesqueros, Instituto de Investigaciones Científicas de la Universidad de Oriente. Boca \\ del Río, isla de Margarita, Venezuela; 1wgc25@gmail.com, neslava20@yahoo.es, franciscogm2002e@hotmail.com \\ 2. Área de Ecología y Ambiente, Instituto de Investigaciones Científicas de la Universidad de Oriente. Boca del Río, isla \\ de Margarita, Venezuela; luis.troccoli@gmail.com
}

Recibido 21-IV-2014. Corregido 01-X-2014. Aceptado 05-XI-2014.

\begin{abstract}
Octopus vulgaris (Octopoda: Octopodidae) biology and fishery on the shores of Nueva Esparta state, Venezuela. In the State of Nueva Esparta, Venezuela, the fishery of octopus Octopus vulgaris is considered an alternative activity by the artisanal fishers to diversify production and to increase their income. Nevertheless, the inter annual fluctuations that have been witnessed in recent years, suggest that this resource requires an urgent analysis of the species reproduction, growth, mortality and the fishery activity, in order to provide a scientific basis to develop sustainable management strategies. For this, weekly samples were collected during the June-December 2012 El Tirano fishing season. Mantle length (ML), total weight (TW), sex and stage of gonadal development were recorded for 1268 males (9-25 cm ML) and 818 females (9-22 cm ML). The monthly sex ratio differed from one, except in June and August, with a high percentage of mature individuals in both sexes. Minimum and average sizes were: $11 \mathrm{~cm} \mathrm{ML}(428 \mathrm{~g})$ and $16 \mathrm{~cm}$ ML $(1142 \mathrm{~g})$ in sexually mature males, and $12 \mathrm{~cm} \mathrm{ML} \mathrm{(476g)} \mathrm{and} 15.35 \mathrm{~cm}$ ML (844g) in sexually mature females. The length-weight relationship of males $\left(\mathrm{TW}=0.7994 * \mathrm{ML}^{2.62}\right.$ ) and females $\left(\mathrm{TW}=1.4552 * \mathrm{ML}^{2.33}\right.$ ) showed minor allometric growth. Growth was estimated by analysis of the frequency distribution of lengths, using FiSAT software. Growth parameters, estimated by the von Bertalanffy model, were considered rapid, being $\mathrm{L}_{\infty}=26.26 \mathrm{~cm}, \mathrm{~W}_{\infty}=3769 \mathrm{~g}, \mathrm{k}=2.3 /$ year, and $\mathrm{t}_{\mathrm{o}}=-0.015 /$ year, in males; and $\mathrm{L}_{\infty}=24.28 \mathrm{~cm}, \mathrm{~W}_{\infty}=2287 \mathrm{~g}, \mathrm{k}=1.8 /$ year, and $\mathrm{t}_{\mathrm{o}}=-0.09 /$ year, in females. The maximum age in males was $\mathrm{A}_{0.95}=1.3$ years and $\mathrm{A}_{0.95}=1.57$ years in females. Octopus captures were directly significant with superficial water temperature, but negatively significant with wind velocity and precipitation, which corresponds to a study zone with seasonal hydrologic variability. The actual exploitation rate $(\mathrm{E}=0.61$ / year in males, $E=0.60 /$ year in females) was calculated by using the values of total mortality $(\mathrm{Z}=7.73 /$ year in males, $\mathrm{Z}=6.63$ /year in females), for fishing ( $\mathrm{F}=4.7 /$ year in males, $\mathrm{F}=3.99 /$ year in females), and natural $(\mathrm{M}=3.03$ / year in males, $M=2.64 /$ year in females), indicating that the octopus is over-exploited. Consequently, we recommend regulating the fishery by reducing the fishing mortality rate. Rev. Biol. Trop. 63 (2): 427-442. Epub 2015 June 01 .
\end{abstract}

Key words: Octopus vulgaris; reproduction; growth; mortality; fishery; Venezuela.

El pulpo común Octopus vulgaris Cuvier 1797 es una especie bentónica con desarrollo continuo, ciclo de vida corta, gran inteligencia motora y agudeza visual. Los huevos son puestos en grupo y son bentónicos. La hembra recubre la puesta con su cuerpo, y de vez en cuando renueva el agua alrededor de los huevos por medio de contracciones del manto y los limpian con los brazos. Huevos de pulpo pueden ser encontrados en cualquier punto de la plataforma continental, donde resida esta especie con fase larvaria planctónica susceptible de ser influenciada por el afloramiento costero (Otero, 2006).

La mayoría de los cefalópodos, responden rápidamente a las variaciones ambientales debido a que sus poblaciones son más susceptibles que otras especies, porque tienen un ciclo 
de vida corto, caracterizado por la seme paridad como adaptación evolutiva para el oportunismo ecológico, lo que le permite a las poblaciones crecer rápidamente cuando las condiciones ambientales son favorables (Rocha, Guerra, \& González, 2001).

De las siete especies del género Octopus reportadas en Venezuela, dos de ellas son de interés comercial: el pulpo común Octopus vulgaris y el pulpo de arrecife Octopus briareus, estimándose que la mayor parte de los desembarques corresponden a Octopus vulgaris (Robaina, 1986). En las costas insulares del estado Nueva Esparta, Venezuela, el pulpo es ampliamente explotado y con importancia socioeconómica, considerada como actividad alternativa para diversificar la producción de los pescadores artesanales, ocupando un lugar importante junto a otros recursos convencionales que a lo largo de los últimos años han venido experimentando fluctuaciones interanuales considerables.

La pesca del pulpo en Venezuela está regulada por normas establecidas en la Gaceta Oficial $\mathrm{N}^{0} 39.017$ de fecha 16 de septiembre de 2008, donde se dispone que los pescadores deberán tener un permiso especial para su captura, y se establece una época de veda a partir del 01 de enero al 30 de junio de cada año; así mismo, se determina un peso mínimo de captura, movilización y extracción de $400 \mathrm{~g}$ de peso.

Ante la imperante necesidad de manejar el recurso de una forma racional y responsable, de manera que su explotación sea sostenible, se plantearon objetivos que conlleven a conocer la dinámica reproductiva y el crecimiento, de modo que sirvan de fundamento biológico; asimismo, analizar su pesquería en uno de los sistemas de afloramiento costero más productivos del país. La justificación de este trabajo radica en sentar las bases científicas sobre las que se ha de apoyar un futuro diseño para llevar a cabo estrategias de gestión de $O$. vulgaris en Venezuela, que ya funciona con éxito en otras especies de pulpo (Pereiro, \& Bravo de Laguna, 1979). Por lo tanto, es necesario regular su aprovechamiento para lograr una producción sostenida. Alcanzar la sostenibilidad de la pesquería requiere transformar los procesos de administración del recurso. Ello pasa por adoptar varias medidas, como la reducción drástica de la presión de pesca para obtener mayores rendimientos; el respeto escrupuloso de las medidas de gestión ya adoptadas e introducir en el ecosistema al pescador como parte del mismo).

\section{MATERIALES Y MÉTODOS}

Los ejemplares de Octopus vulgaris fueron capturados con nasa y potera por la flota pesquera artesanal de El Tirano, que faena en zonas cercanas al archipiélago Los Frailes (11 $\left.{ }^{\circ} 11^{\prime} 30^{\prime \prime} \mathrm{N}-63^{\circ} 42^{\prime} 00^{\prime \prime} \mathrm{W}\right)$, localizado al noreste de la isla de Margarita del estado Nueva Esparta (Fig. 1).

La nasa es una trampa que consiste en una caja de forma hexagonal, dividida en tres partes: las caras o tapas elaboradas con mallas plásticas con abertura de cinco centímetros, los laterales o ruedos confeccionados con tela de malla de alambre galvanizado de cinco centímetros, y la boca que posee forma de embudo elaborada del mismo material que el anterior. Las nasas son lanzadas al mar en "enyugues"que consiste en una línea de dos a diez nasas unidas por una cuerda de polietileno de $3 / 8$ " de grosor con una distancia entre una y otra de 15 a $20 \mathrm{~m}$. Las profundidades donde se colocan las nasas oscilan de 20 a $50 \mathrm{~m}$, y las dejan de 2 a 20 días sin carnada y sin ningún dispositivo de señalización, marcando el lugar con un GPS (González, Eslava, \& Guevara, 2001).

La potera es un arte de pesca conformada por un tubo de acero galvanizado relleno con plomo de $20 \mathrm{~cm}$, que a su vez sirve de peso, en la cual van adheridos cuatro anzuelos en el extremo superior, y dos anzuelos o cuatro alambres de hierro galvanizado encorchado en el tubo por su parte media y cuatro alambres en la parte inferior del mismo. En el extremo libre del alambre va unido un anzuelo, cuyo tamaño varía de acuerdo a la ubicación en el tubo, desde el tamaño de anzuelo número cuatro en la parte superior hasta el tamaño número siete en 


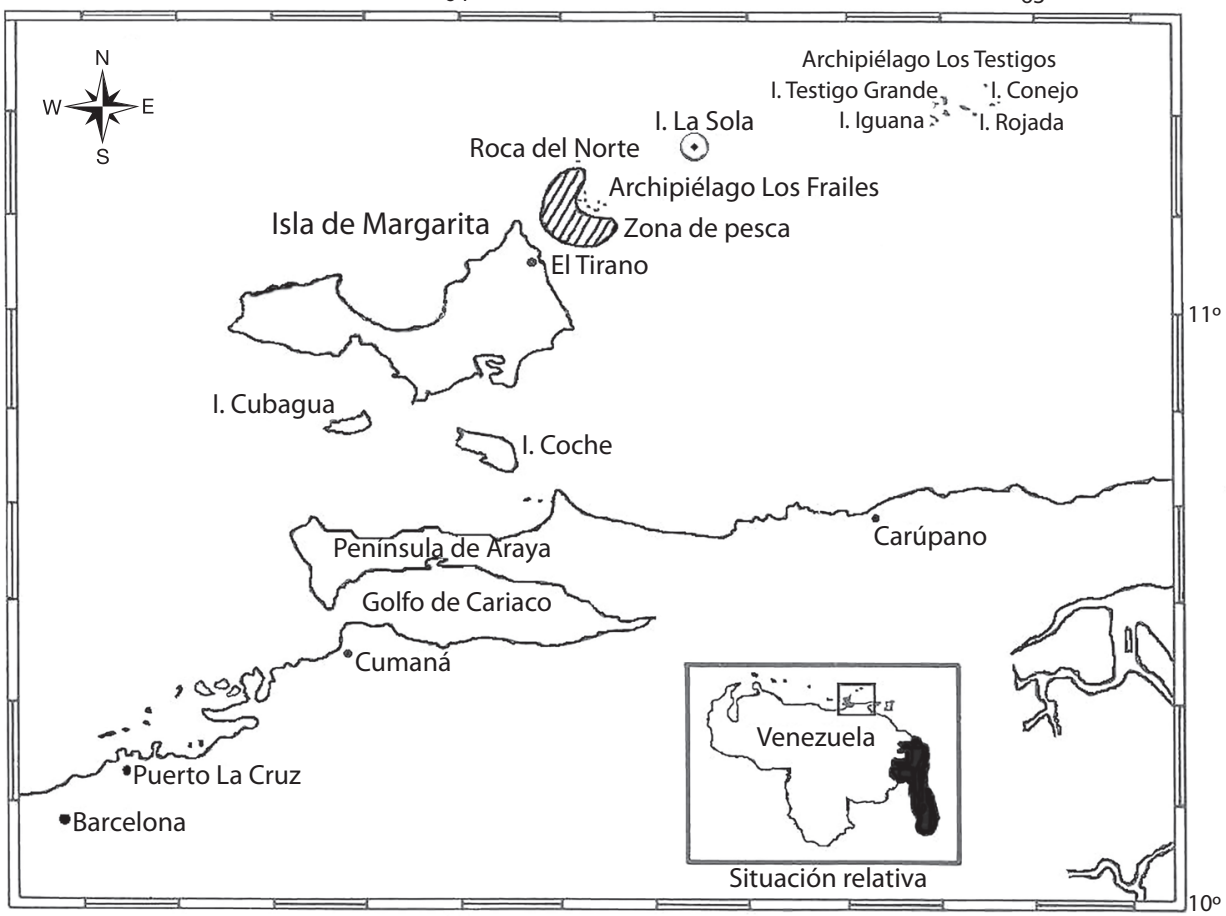

Fig. 1. Zona de pesca de Octopus vulgaris, en la temporada de pesca junio-diciembre 2012, Nueva Esparta, Venezuela. Fig. 1. Fishing zone of Octopus vulgaris, during the June-December 2012 fishing season, Nueva Esparta, Venezuela.

la parte media e inferior, dispuestos en círculos formando una especie de corona. La carnada utilizada es bagre (Bagre marinus) y/o morena (Lycodontis moringa) por su consistencia dura o en su defecto emplean un trapo rojo como señuelo colocado, en cualquiera de los casos, en la parte media o inferior del tubo. Cada pescador opera una o dos poteras que están unidas por su extremo superior a una línea de nylon monofilamento de 18 a $25 \mathrm{~m}$ de longitud, las cuales son arrastradas por espacio de 10 a 20min, aproximadamente, dependiendo de la abundancia del recurso (González et al., 2001).

Los muestreos se realizaron semanalmente durante la temporada de pesca junio-diciembre 2012 en la playa del Puerto El Tirano ubicada en el municipio Antolín del Campo de la isla de Margarita. A cada ejemplar se le midió la longitud del manto ( $\mathrm{Lm}$ ) comprendida entre los ojos y el extremo distal del manto teniendo en cuenta el eje longitudinal dorsal (Guerra, \& Manríquez, 1980), para ello se utilizó una cinta métrica con apreciación de un milímetro. El peso total sin eviscerar $(\mathrm{Pt})$ se obtuvo con una balanza digital, con capacidad de tres kilogramos y apreciación de un gramo, y se identificó el sexo y cuatro estados de madurez mediante la observación del aparato reproductor ubicando los oviductos en hembras y el pene en machos, siguiendo el criterio descrito por Guerra (1975). Para analizar diferencias significativas respecto a la proporción sexual entre machos $(\mathrm{M})$ y hembras $(\mathrm{H})$ se aplicó la prueba chi-cuadrado (Zar, 1996) $(\alpha=0.05$, $\left.\chi^{2}=3.481\right)$ con corrección de Yates para continuidad (Steel, \& Torrie, 1985). La talla media de primera madurez sexual del $50 \%$ de la población $\left(\mathrm{L}_{50}\right)$ se estimó según la ecuación modificada de la curva logística (Hoydal, Rorvick, \& Sparre, 1982): $\mathrm{FM}_{(\mathrm{L})}=1 /\left[1+\mathrm{e}^{(\mathrm{S} 1-\mathrm{S} 2) * \mathrm{~L}}\right]$, donde $\mathrm{FM}_{(\mathrm{L})}$ es la proporción de individuos maduros a cada talla; $\mathrm{S}_{1}$ y $\mathrm{S}_{2}$ son constantes y $\mathrm{L}$ es la marca de clase. Las constantes $\mathrm{S}_{1}$ y $\mathrm{S}_{2}$ se calcularon usando una ecuación de regresión 
lineal, para la cual se tomó en cuenta: $\ln (1 /$ $\left.\mathrm{FM}_{(\mathrm{L})}-1\right)=\mathrm{S}_{1}-\left(\mathrm{S}_{2}{ }^{*} \mathrm{~L}\right) ; \mathrm{L}_{50}=\mathrm{S}_{1} / \mathrm{S}_{2}$, donde, $\mathrm{S}_{1}=\mathrm{a}$ y $\mathrm{S}_{2}=-$ b.

La relación longitud-peso se realizó a través de la ecuación de Ricker (1975): Pt $=\mathrm{a}_{*} \mathrm{Lm}^{\mathrm{b}}$, donde, Pt es el peso total; Lm es la longitud del manto; "a" es la intersección del eje de las ordenadas y "b" es la pendiente de la curva que fue estimada mediante el ajuste de una línea de regresión a los datos previamente transformados logarítmicamente.

La estimación de los parámetros de crecimiento se basó en el análisis de la distribución de frecuencia de las longitudes del manto. Para ello se usó el software FiSAT (Gayanilo, Sparre, \& Pauly, 1996). En primer lugar, se estimó una longitud asintótica $\left(\mathrm{L}_{\infty}\right)$ aplicando el método de Powell (1979) y Wetherall (1986), que permitió determinar el coeficiente de crecimiento (k) a través de la rutina ELEFAN I, previa reestructuración de la frecuencia de longitudes y asumiendo un parámetro de oscilación igual a cero. En segundo lugar, se calculó el $\mathrm{t}_{\mathrm{o}}$ de acuerdo a la fórmula anotada por Pauly (1979): $\log _{10}\left(-\mathrm{t}_{\mathrm{o}}\right)=-0.3922-0.2752 * \log _{10} \mathrm{~L}_{\infty}-$ $1.038 * \log _{10} \mathrm{k}$; donde $\mathrm{t}_{\mathrm{o}}$ es la edad teórica en que el organismo tiene talla cero, $\mathrm{L}_{\infty}$ es la longitud asintótica del manto, $\mathrm{k}$ es el coeficiente de crecimiento o coeficiente de curvatura. Para el cálculo de la longevidad se utilizó la ecuación de Taylor (1958): $\mathrm{A}_{0.95}=\mathrm{t}_{\mathrm{o}}+2.996 / \mathrm{k}$; donde $\mathrm{A}_{0.95}$ es la edad límite o tiempo requerido para alcanzar el $95 \%$ de la $\mathrm{L}_{\infty}$. El peso asintótico $\left(\mathrm{P}_{\infty}\right)$ fue obtenido usando las constantes a y b de la relación longitud-peso y las longitudes asintóticas $\left(\mathrm{L}_{\infty}\right)$ estimadas según Csirke (1980): $\mathrm{P}_{\infty}=\mathrm{a}^{*} \mathrm{~L}_{\infty}{ }^{\mathrm{b}}$; donde $\mathrm{P}_{\infty}$ es el peso asintótico $\mathrm{o}$ peso máximo promedio. Una vez ajustados $\mathrm{L}_{\infty}$, $\mathrm{P}_{\infty}, \mathrm{k} \mathrm{y}_{\mathrm{o}}$, tanto en machos como en hembras, se determinaron las curvas de crecimiento en longitud y en peso adecuadas al modelo de von Bertalanffy (1938), según las siguientes ecuaciones: $\mathrm{Lm}=\mathrm{L}_{\infty}\left[1-\mathrm{e}^{-\mathrm{k}(\mathrm{t}-\mathrm{to})}\right] ; \quad \mathrm{Pt}=\mathrm{P}_{\infty}\left[1-\mathrm{e}^{-\mathrm{k}(\mathrm{t}-}\right.$ to) $]^{\mathrm{b}}$. Las curvas de crecimiento, de longitud del manto para ambos sexos, fueron comparadas a través de un análisis de varianza de doble vía con un nivel de significancia de 0.05 (Sokal, \& Rohlf, 1995).
La mortalidad total (Z) se obtuvo de la curva de captura linearizada. La mortalidad natural (M) se calculó de acuerdo con la ecuación de Pauly (1980): $\log _{10} \mathrm{M}=-0.0066-0.279 *$ Lo$\mathrm{g}_{10} \mathrm{~L}_{\infty}+0.6543 * \log _{10} \mathrm{k}+0.4632 * \log _{10} \mathrm{~T}$, utilizando una temperatura $(\mathrm{T})$ media anual del área de $25^{\circ} \mathrm{C}$. La mortalidad por pesca (F) se logró de la diferencia entre $\mathrm{Z}$ y $\mathrm{M}$, y la tasa de explotación (E) se calculó utilizando la ecuación: $\mathrm{E}=\mathrm{F} / \mathrm{Z}$, suponiendo que el $\mathrm{E}_{\text {optimo }}=0.5$ (Gulland, 1971), que significa una población en estado de plena explotación, donde mueren igual número de individuos por muerte natural que por explotación pesquera.

Los datos de captura del pulpo fueron proporcionados por el Instituto Socialista de Pesca y Acuicultura (INSOPESCA), y para establecer posibles relaciones entre las capturas y las variables ambientales, se realizó un Análisis de Componentes Principales a partir de la matriz de correlación (Johnson, \& Wichern, 1992), los datos de Temperatura Superficial del Mar (TSM) fueron obtenidos a partir de la serie de tiempo del proyecto CARIACO (USF-IMARS), la Velocidad del Viento (VV) y la Precipitación (P) del Servicio Meteorológico de la Fuerza Aérea Venezolana de la Estación de Porlamar.

\section{RESULTADOS}

Se registró un total de 2086 individuos de Octopus vulgaris; 1268 machos y 818 hembras. La talla osciló en machos de 9 a $25 \mathrm{~cm}$ con un promedio de $16.95 \mathrm{~cm}( \pm 2.62)$ y en hembras de 9 a $23 \mathrm{~cm}$ con un promedio de $15.25 \mathrm{~cm}$ $( \pm 2.51)$. En tanto que el peso varió en machos de 193 a $3818 \mathrm{~g}$ con un promedio de $1424.32 \mathrm{~g}$ $( \pm 616.66)$ y en hembras de 240 a $2499 \mathrm{~g}$ con un promedio de $875.38 \mathrm{~g}( \pm 397.52)$ (Cuadro 1).

Distribución de tallas: Octopus vulgaris ingresa a la zona de pesca a partir de una talla de $9 \mathrm{~cm}$ alcanzando los machos mayores tallas que las hembras. Las tallas fluctuaron mensualmente en machos de 14 a $19 \mathrm{~cm}$, siendo el de mayor abundancia el de $18 \mathrm{~cm}$; y en hembras de 11 a $16 \mathrm{~cm}$ con un pico de abundancia de $16 \mathrm{~cm}$. La mayor cantidad se observó en el periodo julio-septiembre en ambos sexos (Fig. 2). 


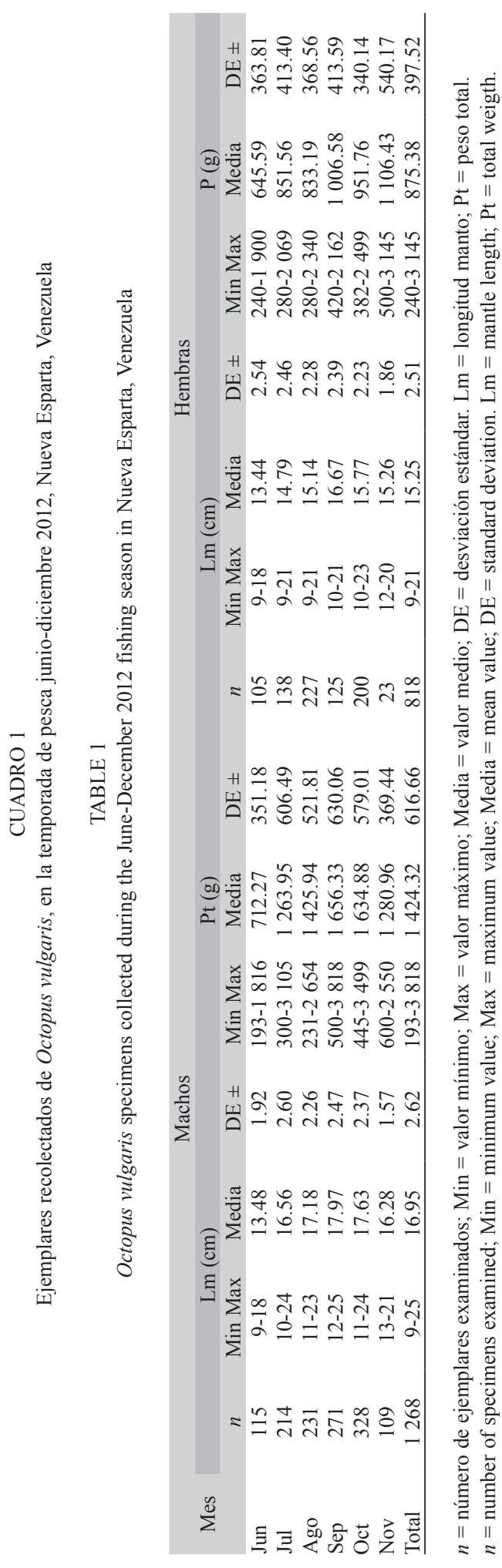

Reproducción: La proporción de sexos fue significativamente diferente de uno durante la mayor parte de la temporada de pesca, excepto junio y agosto; sin embargo, para la totalidad del período de estudio la proporción sexual global fue 1.6 machos y 1.0 hembras $\left(\chi^{2}=96.64, p<0.05\right)$ (Cuadro 2). Sobre la escala de madurez empleada, se observó que los machos presentan condiciones aptas para la reproducción definidas por la presencia de organismos en estadio III a lo largo de toda la temporada de pesca. No obstante, la tendencia general indica que noviembre es de mayor actividad en los machos, con un marcado descenso en septiembre. En las hembras por su parte, la ausencia del estadio IV en junio-julio y noviembre, supone un breve período en que éstas alcanzan la condición apta para la reproducción; coincidiendo con la mayor proporción de machos prestos a este evento (Cuadro 2). En términos generales, durante la temporada de pesca junio-diciembre 2012, los machos y las hembras maduros presentaron el más alto porcentaje con $77.83 \%$ y $77.63 \%$, respectivamente, respecto a los otros estados de madurez sexual (Machos: Inmaduros 1.11\%, Madurando $2.37 \%$, Desovados 18.69\%; Hembras: Inmaduras $4.77 \%$, Madurando $9.41 \%$, Desovadas $8.19 \%$ ). Las talla mínima de madurez sexual en los machos fue de $11 \mathrm{~cm}$ con un peso de $428 \mathrm{~g}$ y la talla media del $50 \%$ de la población fue de $16 \mathrm{~cm}$ con un peso de $1142 \mathrm{~g}$; mientras que en las hembras fue de $12 \mathrm{~cm}$ la mínima con un peso de $476 \mathrm{~g}$ y la media del $50 \%$ fue de $15.35 \mathrm{~cm}$ con un peso de $844 \mathrm{~g}$ (Fig. 3).

Relación longitud-peso: Las longitudes medias de machos y hembras presentaron diferencias significativas $\left(t_{\mathrm{s}}=7.76, \mathrm{p}<0.05\right)$, igualmente en la relación longitud-peso los valores de la pendiente $\mathrm{b}\left(\mathrm{t}_{\mathrm{s}}=5.09, \mathrm{p}<0.05\right)$ e intersección a $\left(\mathrm{t}_{\mathrm{s}}=11.51, \mathrm{p}<0.05\right)$; razón por la cual la expresión se estableció por separado, quedando en los machos: $\mathrm{Pt}=0.7994 * \mathrm{Lm}^{2.62}$ y en las hembras: $\mathrm{Pt}=1.4552 * \mathrm{Lm}^{2.33}$ (Fig. 4 ).

Crecimiento: Los parámetros de crecimiento en machos fueron: $\mathrm{L}_{\infty}=26.26 \mathrm{~cm}, \mathrm{k}=2.3$ / 

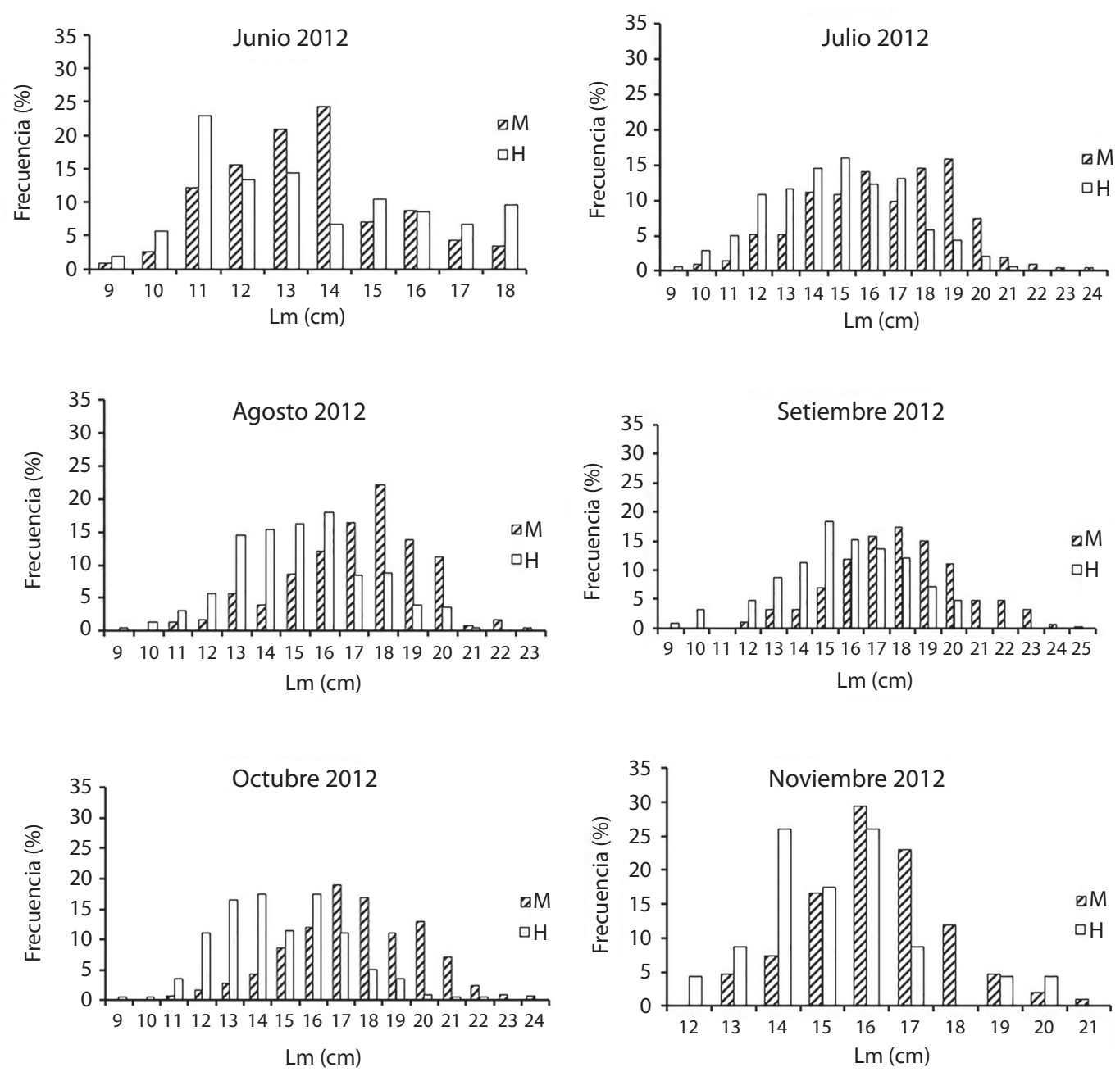

Fig. 2. Distribución mensual de frecuencia de longitudes para machos y hembras de Octopus vulgaris, en la temporada de pesca junio-diciembre 2012, Nueva Esparta, Venezuela.

Fig. 2. Monthly frequency distribution of lengths for male and female Octopus vulgaris, during the June-December 2012 fishing season, Nueva Esparta, Venezuela.

año, $t_{0}=-0.015 /$ año $y$ en hembras fueron: $\mathrm{L}_{\infty}=24.28 \mathrm{~cm}, \mathrm{k}=1.8 / \mathrm{año}, \mathrm{t}_{\mathrm{o}}=-0.09 / \mathrm{año}$. La longevidad o edad límite teórica $\left(\mathrm{A}_{0.95}\right)$ calculada para machos fue de 1.3 años y de 1.57 años en hembras. Las curvas de crecimiento en longitud de machos y hembras (Fig. 5A) ajustadas al modelo de von Bertalanffy describieron curvas de tipo exponencial; observándose un rápido crecimiento hasta 0.5 año de vida, para luego disminuir a medida que alcanza la longitud asintótica. Los pesos asintóticos $\left(\mathrm{P}_{\infty}\right)$ calculados fueron: $\mathrm{P}_{\infty}=5199 \mathrm{~g}$ en machos y $\mathrm{P}_{\infty}=2457 \mathrm{~g}$ en hembras, los cuales fueron reemplazados en la ecuación de crecimiento en peso del modelo de von Bertalanffy describiendo unas curvas del tipo sigmoideo, donde hasta 0.5 años el incremento en peso es lento, para luego ir acelerando, en seguida se produce una inflexión y el crecimiento se va haciendo cada vez más lento acercándose asintóticamente a su peso máximo (Fig. 5B).

Mortalidad: La mortalidad natural establecida para $O$. vulgaris fue $\mathrm{M}=3.03$ /año en 


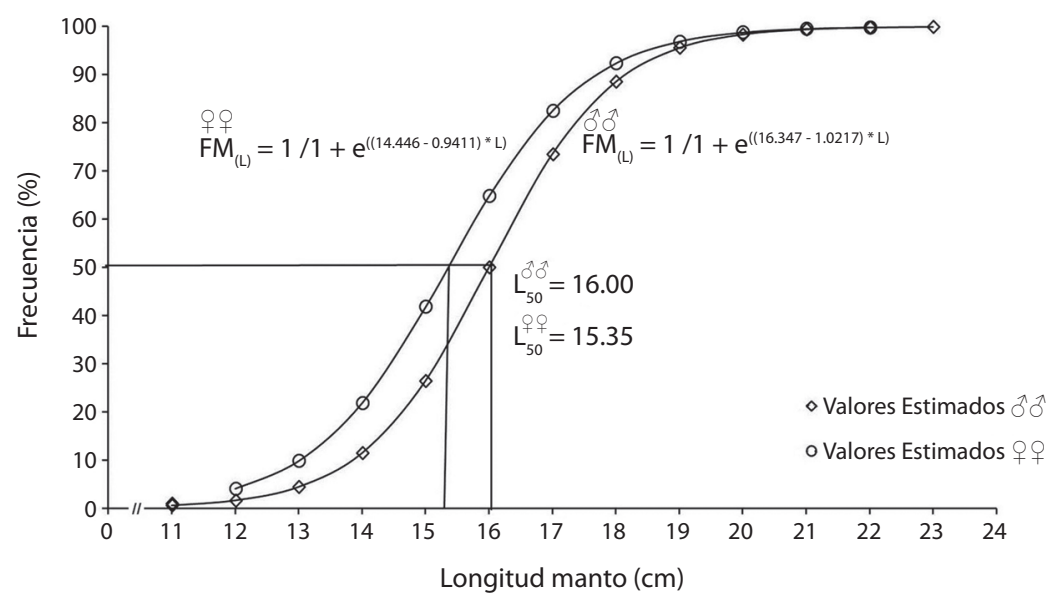

Fig. 3. Longitud media de madurez sexual $\left(\mathrm{L}_{50}\right)$ de Octopus vulgaris machos y hembras, en la temporada de pesca juniodiciembre 2012, Nueva Esparta, Venezuela.

Fig. 3. Mean length at sexual maturity $\left(\mathrm{L}_{50}\right)$ of male and female Octopus vulgaris, during the June-December 2012 fishing season, Nueva Esparta, Venezuela.

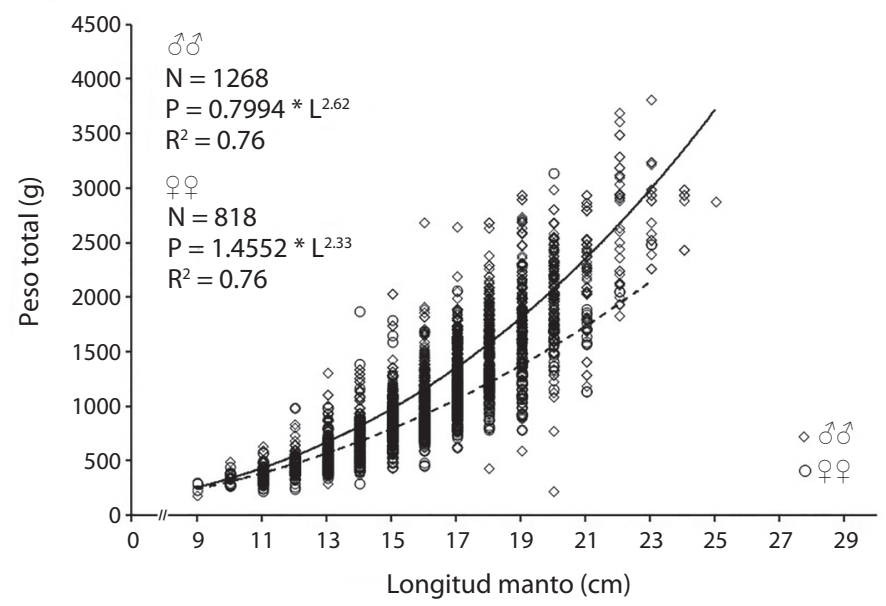

Fig. 4. Relación longitud-peso de Octopus vulgaris machos y hembras, en la temporada de pesca junio-diciembre 2012, Nueva Esparta, Venezuela.

Fig. 4. Length-weight relationship of male and female Octopus vulgaris, during the June-December 2012 fishing season, Nueva Esparta, Venezuela.

machos; M=2.64/año en hembras y la mortalidad total fue $\mathrm{Z}=7.73$ /año en machos (Fig. 6A); $Z=6.63 /$ año en hembras (Fig. 6B). Con las mortalidades, natural y total estimadas, se pudo determinar la mortalidad por pesca $\mathrm{F}=4.7 / \mathrm{año}$ en machos; $\mathrm{F}=3.99 /$ año en hembras y la tasa de explotación actual $\mathrm{E}=0.61 /$ año en machos; $\mathrm{E}=0.60 /$ año en hembras.
Descripción de la pesquería: Las embarcaciones utilizadas para la pesca de pulpo son de madera de seis a siete metros de eslora denominados "peñeros" equipadas con un motor fuera de borda de 40 caballos de fuerza, participando de dos a tres pescadores por embarcación. Los principales artes de captura del pulpo son la nasa del tipo antillana y la potera. 
CUADRO 2

Proporción sexual mensual de Octopus vulgaris en la temporada de pesca junio-diciembre, Nueva Esparta, Venezuela

TABLE 2

Monthly sex ratio of Octopus vulgarisduring the June-December 2012 fishing season in Nueva Esparta, Venezuela

\begin{tabular}{lcccccc}
\multicolumn{1}{c}{ Mes } & Machos & Hembras & Total & Machos:Hembras & $\chi^{2}$ & Significancia \\
Junio & 115 & 105 & 220 & $1.1: 1.0$ & 0.37 & $\mathrm{p}>0.05$ \\
Julio & 214 & 138 & 352 & $1.6: 1.0$ & 15.98 & $\mathrm{p}<0.05^{*}$ \\
Agosto & 231 & 227 & 458 & $1.0: 1.0$ & 0.02 & $\mathrm{p}>0.05$ \\
Septiembre & 271 & 125 & 396 & $2.2: 1.0$ & 52.23 & $\mathrm{p}<0.05^{*}$ \\
Octubre & 328 & 200 & 528 & $1.6: 1.0$ & 30.55 & $\mathrm{p}<0.05^{*}$ \\
Noviembre & 109 & 23 & 132 & $4.7: 1.0$ & 54.73 & $\mathrm{p}<0.05^{*}$ \\
Total & 1268 & 818 & 2068 & $1.6: 1.0$ & 96.64 & $\mathrm{p}<0.05^{*}$ \\
\hline
\end{tabular}

*Proporción sexual significativamente diferente de $1: 1\left(\mathrm{p}<0.05, \chi^{2}=3.841\right)$.

*Sex ratio significantly different $1: 1\left(\mathrm{p}<0.05, \chi^{2}=3.841\right)$.
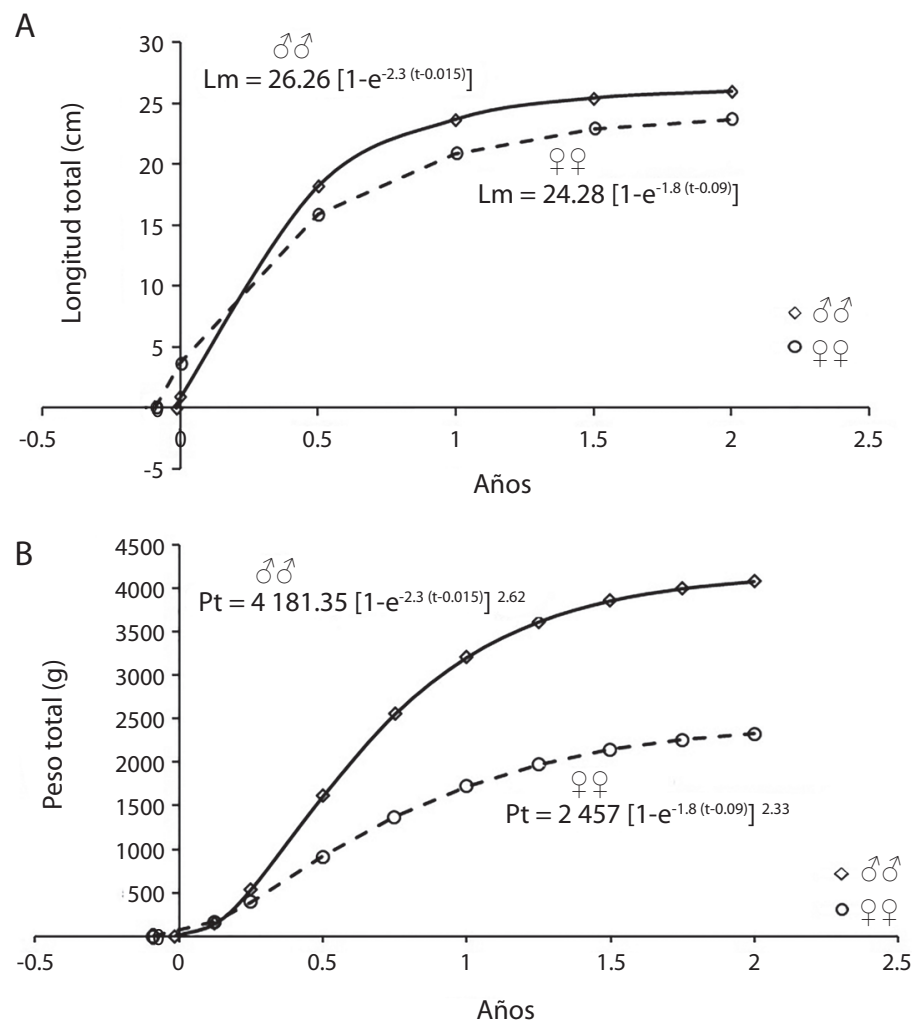

Fig. 5. Curva de crecimiento en longitud (A) y peso (B) de machos y hembras de Octopus vulgaris ajustadas al modelo de von Bertalanffy (1938), en la temporada de pesca junio-diciembre 2012, Nueva Esparta, Venezuela.

Fig. 5. Growth curve in length (A) and weight (B) of male and female Octopus vulgaris, according to the von Bertalanffy model (1938), during the June-December 2012 fishing season, Nueva Esparta, Venezuela. 
A

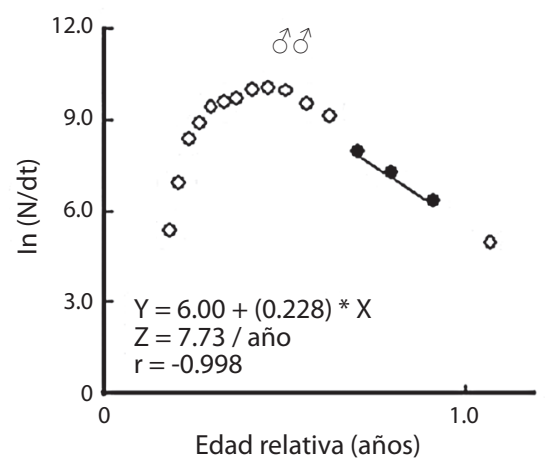

B

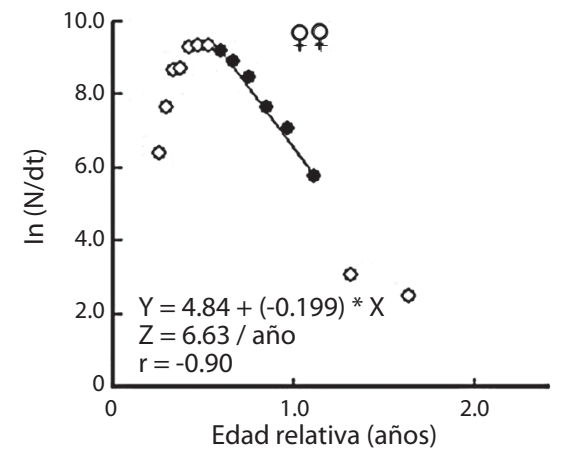

Fig. 6. Estimación de la mortalidad total a partir del método de la curva de captura linearizada de Octopus vulgaris machos (A) y hembras (B), en la temporada de pesca junio-diciembre 2012, Nueva Esparta, Venezuela.

Fig. 6. Estimation of total mortality from a linearized catch curve method of male (A) and female (B) Octopus vulgaris, during the June-December 2012 fishing season, Nueva Esparta, Venezuela.

Capturas: La serie histórica de captura del O. vulgaris 1996-2012 en los estados Sucre y Nueva Esparta mostró fuerte variación interanual, siendo este último estado el de mayor producción, destacando con los mayores volúmenes de captura del 2007 al 2009; mientras que 2001, 2003 y 2012 fueron los de más bajo rendimiento (Fig. 7). Durante la temporada de pesca 2012 en Nueva Esparta también se observó oscilación de las capturas mensuales, siendo agosto (61630kg) y septiembre $(99018 \mathrm{~kg})$ los que presentaron los mayores pulsos y los más bajos en octubre $(16331 \mathrm{~kg})$, noviembre $(19421 \mathrm{~kg})$ y diciembre $(18461 \mathrm{~kg})$; siendo la captura total de $294911 \mathrm{~kg}$ en 567 permisos de pesca otorgados a igual número de pescadores.

Relación recurso-ambiente: Durante la temporada de pesca junio-diciembre 2012 la temperatura superficial del mar (TSM) promedio mensual fluctuó entre 25.9 y $28.8^{\circ} \mathrm{C}$, la velocidad del viento (VV) de 3.15 a $6.59 \mathrm{~m} / \mathrm{s}$ y las precipitaciones $(\mathrm{P})$ desde 0 hasta $87.6 \mathrm{~mm}$. Los valores mensuales de la TSM tuvieron

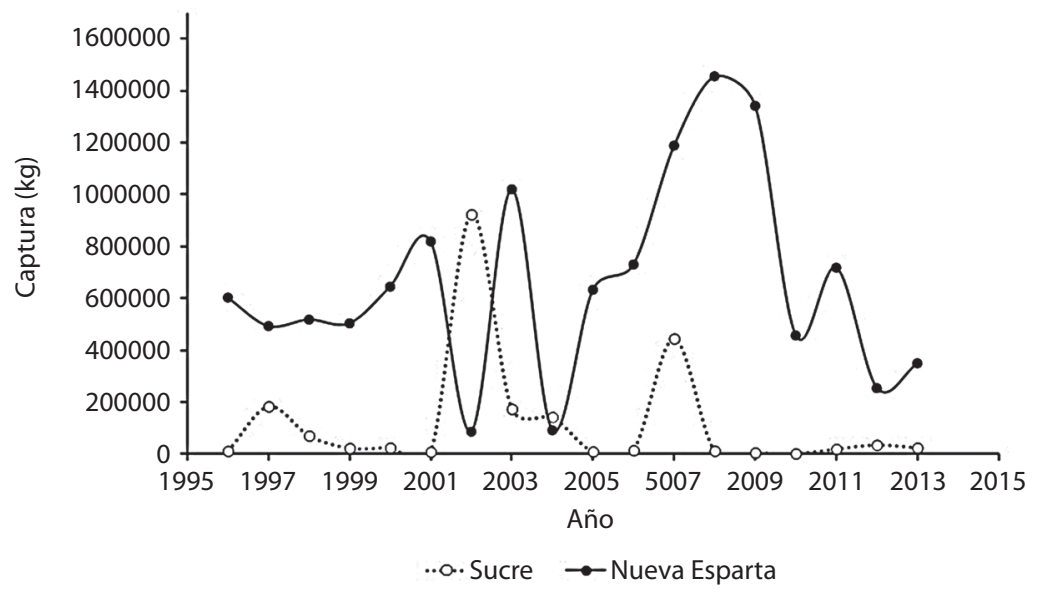

Fig. 7. Capturas de Octopus vulgaris en Sucre y Nueva Esparta, Venezuela, durante 1996-2012. Fuente de datos: INSOPESCA.

Fig. 7. Captures of Octopus vulgaris in Sucre and Nueva Esparta, Venezuela, during 1996-2012. Data source: INSOPESCA. 
una tendencia ascendente desde junio hasta noviembre y se observó un descenso brusco en diciembre, mientras que el descenso de la VV fue sostenido desde junio hasta diciembre, en tanto que la P presentó valores bajos con excepción del mes de octubre que fue alto (Cuadro 3). El análisis de componentes principales mostró una varianza acumulada de $93 \%$ en los dos primeros componentes. El componente I, mostró la relación significativa y positiva entre la captura y la temperatura superficial del mar. Ambas en sentido negativo con la velocidad del viento y precipitación (Fig. 8).

\section{DISCUSIÓN}

La baja proporción de organismos pequeños de nueve centímetros en las capturas puede

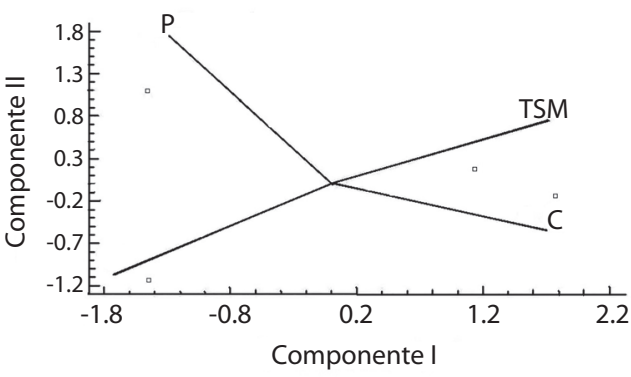

Fig. 8. Análisis de Componentes Principales: Valores del primer y segundo componente principal. Captura de Octopus vulgaris (C), temperatura superficial del mar (TSM), velocidad del viento (VV) y precipitación (P), en la temporada de pesca junio-diciembre 2012, Nueva Esparta, Venezuela.

Fig. 8. Principal Components Analysis: Eigen values for the first and second main components. Captures of Octopus vulgaris (C), superficial water temperature (TSM), wind velocity (VV) and precipitation (P), during the JuneDecember 2012 fishing season, Nueva Esparta, Venezuela.

\section{CUADRO 3}

Variación mensual de capturas de Octopus vulgaris, temperatura superficial del mar (TSM), velocidad del viento (VV) y precipitación (P), en la temporada de pesca junio-diciembre 2012, Nueva Esparta, Venezuela

\section{TABLE 3}

Monthly variation of Octopus vulgaris captures, superficial water temperature, wind velocity, and precipitation, during the June-December 2012 fishing season, Nueva Esparta, Venezuela

\begin{tabular}{lcccc} 
& Captura $(\mathrm{kg})$ & TSM $\left({ }^{\circ} \mathrm{C}\right)$ & VV $(\mathrm{m} / \mathrm{s})$ & $\mathrm{P}(\mathrm{mm})$ \\
Junio & 45850 & 26.2 & 6.59 & 2.0 \\
Julio & 34200 & 26.8 & 5.37 & 15.0 \\
Agosto & 61630 & 28.2 & 4.43 & 0.0 \\
Septiembre & 99018 & 27.8 & 4.06 & 0.0 \\
Octubre & 16331 & 28.5 & 3.51 & 87.6 \\
Noviembre & 19421 & 28.8 & 3.67 & 21.8 \\
Diciembre & 18461 & 25.9 & 3.15 & 27.4 \\
\hline
\end{tabular}

ser resultado de la baja disponibilidad de tallas menores en el área de pesca, lo que significaría que las áreas de reclutamiento corresponderían a espacios que no ocupa la pesquería. No se cuantificó la incertidumbre asociada a la estructura de tallas, porque la fluctuación demanda especial importancia en recursos tropicales, donde ciertas características del ciclo de vida (e.g., reproducción y reclutamiento continuos, y que se producen en áreas diferentes) impiden una clara progresión de clases modales, lo cual resalta la importancia de determinar la influencia que ejerce cada muestra mensual. Es muy común además la presencia de errores de medición y sesgos en la obtención de los datos. En $O$. vulgaris existe una fuente adicional de incertidumbre dada por el acceso a información de capturas comerciales solamente para los seis meses (01 de julio a 31 de diciembre) en los cuales opera la pesquería, como resultado de una medida de manejo.

La abundancia de machos con respecto a hembras fue significativamente alta durante la temporada de pesca, excepto junio y agosto. Esta evidencia puede ser causada, probablemente porque las hembras se refugian en cuevas 
para continuar con su proceso reproductivo, mientras que los machos se quedan en las mismas zonas (conchales) para alimentarse, siendo más vulnerables a ser capturados, y/o por diversos factores, tales como el agrupamiento natural por talla y sexo, el comportamiento, las diferentes tasas de crecimiento entre sexos, el desove y la mortalidad post desove (RodríguezRúa, Pozuelo, Prado, Gómez, \& Bruzón, 2005). Sin embargo, Jiménez-Badillo, del Río-Rodríguez, Gómez-Solano, Cu-Escamilla \& Méndez-Aguilar (2008) no encontraron diferencias significativas en la proporción de machos y hembras de $O$. vulgaris en una población capturada en el Golfo de México.

La mayor abundancia de machos maduros ocurrió en julio y noviembre, mientras que la de hembras en agosto y septiembre, probablemente relacionado con la menor abundancia de machos y hembras en estado de pre-desove y desove. Es una característica común en muchos cefalópodos que los machos maduren primero que las hembras, lo que se considera como una estrategia para el apareamiento (Boyle, \& Rodhouse, 2005). En cefalópodos, la presencia de espermatecas permite a las hembras almacenar espermatóforos de los machos por períodos prolongados antes de la fecundación y el desove (Froesch, \& Marthy, 1975; Fernández-Núñez, Hernández-González, Raya, \& Balgueiúas, 1996; Rocha, 2003); lo cual se asocia con factores intrínsecos de la especie así como con las condiciones ambientales. Aunque no se han muestreado ejemplares en la época de veda, se sospecha que los machos maduros deben estar presentes durante todo el año, los mismos que alcanzan la madurez antes que las hembras para garantizar la disponibilidad de gametos para el proceso reproductivo; tal como lo han sugerido Rodríguez-Rúa et al. (2005). La maduración y desove de $O$. vulgaris en el Atlántico ocurre todo el año en dos periodos, uno intenso de enero a julio con un pico en abril, y otro menos intenso de octubre a noviembre (Guerra, 1992). De esta manera, la presencia de machos en estadio IV en septiembre-octubre y de hembras en octubre con valores altos, después del estadio III, podría significar que en la temporada de pesca una porción de la población está reproduciéndose y la otra en la época de veda, dando origen a dos cohortes independientes por año con ciclos vitales separados en el tiempo.

Las tallas medias de madurez sexual estimadas fueron similares a las obtenidas por Cotúa (2012): machos $=15.41 \mathrm{~cm}$ y $16.08 \mathrm{~cm}$ $\mathrm{Lm}$; hembras $=15.06 \mathrm{~cm}$ y $14.95 \mathrm{~cm} \mathrm{Lm}$ en las temporadas 2010 y 2011, respectivamente, para la misma zona de pesca, pero mayores a las calculadas por Hernández-García, Hernández-López \& Castro-Hernández (2002) en las costas de las Islas Canarias (machos $=10.5 \mathrm{~cm}$ $\mathrm{y}$ hembras $=11.3 \mathrm{~cm} \mathrm{Lm}$ ). Este indicador reproductivo sugiere aumentar el peso mínimo regulado de captura de $400 \mathrm{~g}$ vigente en Venezuela para $O$. vulgaris, porque el peso que corresponde a las tallas medias de madurez sexual determinadas corresponden a $1142 \mathrm{~g}$ en machos y $844 \mathrm{~g}$ en hembras.

Por otra parte, se ha reportado que la reproducción de los octópodos está íntimamente relacionada con la temperatura del agua, la disponibilidad de luz y la alimentación (van Heukelem, 1979; Mangold, 1983; Forsythe, \& van Heukelem, 1987; Mangold, 1987; Roper, Sweeney, \& Huchberg, 1995; Iglesias, Sánchez, \& Otero, 1997; Quetglas, Alemany, Carbonell, Merella, \& Sánchez, 1998). Nuestros resultados muestran que de julio a noviembre, cuando la TSM tiende a incrementarse, se presentan machos y hembras maduros en las capturas artesanales. Es posible que $O$. vulgaris migre hacia áreas menos profundas de las plataformas insulares con el fin de colocar las masas de huevos sobre sustratos rocosos y probablemente en busca de condiciones más adecuadas que las que encuentra a profundidades mayores. Durante la temporada de veda (enero-junio) es cuando se registra la mayor productividad primaria en la región nororiental de Venezuela (Okuda, 1974), por lo que los pulpos recién eclosionados encuentran condiciones adecuadas, para su desarrollo con concentraciones suficientes de alimento, así como temperaturas adecuadas, a profundidades mayores. Durante la temporada reproductiva se presenta un alto 
porcentaje de hembras ovadas en las capturas artesanales, lo que pone en riesgo la tasa de supervivencia de los huevos y por lo tanto, la abundancia del recurso.

El valor de $b$, para machos $(b=2.62)$ y hembras $(b=2.33)$, indicó un crecimiento alométrico minorante, lo cual significa que, a medida que los individuos crecen se modifican sus proporciones corporales, siendo, en este caso, menos pesados para la longitud que tiene. Dichos resultados coinciden con los reportados por Cotúa (2012) en el archipiélago Los Frailes durante las temporadas de pesca 2010 (machos: $b=2.49$; hembras: $b=2.04$ ) y 2011 (machos: $b=2.70$; hembras: $b=2.47$ ) y con Otero (2006) en el litoral gallego (machos: $b=2.00$; hembras: $b=2.11$ ). Este tipo de crecimiento sugiere que hay mayor gasto energético en la temporada reproductiva. La relación longitud-peso mostró que los machos alcanzan mayores tallas y pesos que las hembras. Esto en los octópodos, parece ser una generalidad asociada con el proceso reproductivo debido a que las hembras detienen su crecimiento para destinar gran parte de la energía a la reproducción, mientras que los machos continúan creciendo aún después de alcanzar la madurez sexual. El mayor peso de los machos, también, puede ser explicado por el dimorfismo sexual, en cuanto al peso, los machos son más pesados que las hembras. Este factor conllevaría a considerar que los resultados obtenidos en la presente investigación corroboran las características biológicas de la especie. En las costas de Islas Canarias, HernándezGarcía et al. (2002) obtuvieron valores mayores de 3 (machos: $b=3.09$; hembras: $b=3.11$ ).

Las longitudes asintóticas y coeficientes de curvatura estimadas de $O$. vulgaris en este estudio fueron diferentes al obtenido por Pereiro \& Bravo de Laguna (1979) en el Atlántico Centro Oriental para machos $\left(\mathrm{L}_{\infty}=32.51 \mathrm{~cm}\right.$; $\mathrm{k}=0.38 / \mathrm{año})$ y hembras $\left(\mathrm{L}_{\infty}=24.5 \mathrm{~cm} ; \mathrm{k}=0.51\right.$ / año). En tal sentido, se puede considerar que las diferencias detectadas en la misma especie son debidas, posiblemente, a las zonas geográficas, a la aplicación de métodos distintos en la determinación de edad y crecimiento, y a la disparidad en el diseño de muestreo. El índice de curvatura estimado para ambos sexos, indica un crecimiento moderadamente rápido en machos y rápido en hembras, esta característica pudiera tener una respuesta fisiológica explicada por la relación alométrica existente entre el índice metabólico y el peso corporal, dado el ciclo de vida planctónica en su fase larvaria y bentónica en su estado adulto.

La diferencia de la edad límite en machos y hembras puede ser atribuible al mayor valor de $\mathrm{k}$ de las hembras quienes alcanzan la longitud asintótica en menor tiempo que los machos, lo que indica que el crecimiento de los machos es más lento y por consiguiente tienen un ciclo de vida más largo para lograr alcanzar la longitud asintótica. Al mismo tiempo, el hecho de que los machos presenten mayor longevidad podría estar relacionado con el comportamiento de las hembras; aunque no existe una relación precisa entre talla y edad para esta especie, pueden alcanzar de 12 a 18 meses de longevidad en aguas templadas (Mangold, \& Boletzky, 1973). Muchas discrepancias ha despertado la longevidad, debido a las dificultades de la determinación de su edad. Se ha atribuido una edad máxima para machos y hembras de un ciclo biológico próximo a un año en la costa noroccidental africana (Perales, 2001); mientras que Pereiro \& Bravo de Laguna (1979) afirman que en el noroeste africano alcanzan hasta dos años de longevidad, pudiendo los machos lograr $2500 \mathrm{~g}$ en 15 meses de vida mientras que las hembras los $2000 \mathrm{~g}$ a esa misma edad. Las estimaciones de crecimiento de $O$. vulgaris coinciden además con aspectos claves de su biología, tal como su corta longevidad, estimada en este trabajo.

Los valores calculados de las mortalidades de $O$. vulgaris fueron altas porque el FiSAT siempre proporciona sobre estimaciones de estos parámetros; sin embargo, la fase larval planctónica y el canibalismo acentuado en los periodos próximos a la reproducción y en especial entre hembras (Hernández-López, 2000), probablemente, sean unas de las causas de alta mortalidad natural, considerándose siempre como un factor constante que afecta por igual y en forma permanente, pero puede variar con el crecimiento 
de la cohorte y entre cohortes en función de las variables ambientales como la temperatura del agua, la naturaleza de la especie y su vocación ontogenética (Marco Espino 2014, com. pers.); mientras que la mortalidad por pesca no siempre puede ser considerada como una constante, ya que en gran parte está sujeta a la presión de pesca ejercida por los deseos o habilidades del hombre (Csirke, 1980). Una alta tasa de mortalidad total, también, sugiere un fuerte impacto de la pesca, cuyo efecto directo puede causar cambios en la abundancia, en relación a un ecosistema equilibrado, y también en la estructura de tallas, con una reducción en las tallas máximas (Sparre, \& Venema, 1997). Valores de mortalidad menores a los estimados en otras regiones, como el Atlántico Centro Oriental (Pereiro, \& Bravo de Laguna, 1980), puede suponer una condición saludable para la especie. El valor de la tasa de explotación (E) obtenido en el presente trabajo fue alto, por lo que se establece que esta especie esta sobreexplotada. La tasa de explotación es un parámetro que permite conocer cuál es la situación de un stock respecto a la presión de pesca. Cada especie tiene una tasa de explotación máxima en donde no hay interferencia con la reproducción. Pasado el límite los mecanismos compensatorios no permiten que la población se recupere (Tresierra, \& Culquichicón, 1993). Por otra parte, Gulland (1971) menciona que la tasa de explotación óptima ( $\left.\mathrm{E}_{\text {óptima }}\right)$ se da cuando $\mathrm{F}=\mathrm{M}$, de manera general se considera que $\mathrm{E}_{\text {optima }} \approx 0.5$.

Aunque la captura total fue una de las más bajas de los últimos siete años no es un indicador científico de la abundancia, ni existe evidencia de inestabilidad en la dinámica reproductiva y crecimiento del pulpo por la presión de pesca. Sucede simplemente que, en ciertas circunstancias ambientales, como fuertes corrientes de fondo, el recurso no está disponible a la pesca (Juan Miguel Rodríguez 2014, com. pers.). Los cefalópodos son organismos resilientes con capacidad para absorber perturbaciones, sin alterar significativamente sus características de estructura y funcionalidad, pudiendo regresar a su estado original una vez que la perturbación ha terminado, debido al rápido crecimiento, edad temprana de madurez sexual, corto periodo de vida (Hernández-Sánchez, \& De Jesús-Navarrete, 2010) y alta tasa de supervivencia larval y post larval permitiendo que su biomasa se renueve casi en su totalidad de una generación a la siguiente, a diferencia de $O$. maya con desarrollo directo y sin fases larvales posteriores a la eclosión del huevo (Warnke, 1999). No obstante, para O. vulgaris, donde el ciclo biológico es muy breve que contempla una fase planctónica, debe tenerse muy en cuenta la variabilidad ambiental como factor coadyuvante. Las variaciones ambientales pueden afectar algunos aspectos del ciclo de vida de los pulpos, como la tasa de alimentación, crecimiento y maduración, así como la distribución y abundancia de los mismos; por lo tanto la influencia de las variables ambientales sobre los pulpos es un factor importante que debe ser considerado al momento de realizar evaluaciones poblacionales y a la hora de tomar medidas que regulen su pesquería (Katsanevakis, \& Verriopoulos, 2004).

EL ACP mostró correlación positiva entre la captura y la TSM como consecuencia de la respuesta del comportamiento natural del pulpo al incremento de la temperatura que ocurrió durante la temporada de pesca 2012. En el área nororiental de Venezuela la surgencia costera ocurre durante el período enero-abril, y luego comienza el incremento de la temperatura como consecuencia de la disminución de la velocidad del viento (Muller-Karger, \& Aparicio, 1994). Por ello, se sugiere que a partir de julio, hay mayor disponibilidad de alimento (Otero et al., 2008) y la menor turbulencia en el hábitat del pulpo, lo que permite su mayor actividad, siendo más susceptibles a las capturas. Al respecto, coinciden Demarcq \& Faure (2000) en que la captura de la especie se incrementa en el período de estratificación de las aguas. Las altas precipitaciones de octubre no permitieron mayores capturas en el último trimestre del año, debido a que las lluvias arrastran sedimentos, material vegetal y orgánico hacía el mar, provocando un enturbiamiento de las aguas costeras, posiblemente este evento provocó que los pulpos migraran hacia zonas 
más profundas, quedando poco disponibles para la pesca artesanal. Es difícil establecer con precisión la influencia de la variabilidad ambiental anual en la biología del pulpo, esto posiblemente se deba a la surgencia costera y el efecto del río Orinoco que se alternan en sus máximos estacionales, induciendo una alta productividad sostenida todo el año, esto al menos sobre la plataforma continental (González, Euán, Eslava, \& Suniaga, 2007).

Los efectos de la pesca y una inadecuada regulación, probablemente tengan un impacto negativo sobre sus poblaciones. Un modelo conceptual de la pesquería artesanal de pulpo, permitiría disponer de una herramienta de análisis que facilitaría evaluar el comportamiento de la pesquería desde una perspectiva biológica, social y económica. Esta herramienta podría ser empleada en el análisis del potencial bioeconómico dinámico de estrategias de manejo, considerando los diferentes subsistemas que la integran.

Se concluye que el pulpo Octopus vulgaris de las costas de Nueva Esparta, Venezuela, presenta crecimiento diferencial de machos y hembras, con altos porcentajes de individuos maduros durante la temporada de pesca. Es una especie de crecimiento rápido y vida corta con tasas de mortalidad alta, y su captura responde positivamente con la temperatura superficial del mar como consecuencia de respuesta del comportamiento natural del pulpo al incremento de la temperatura, y negativa con la velocidad del viento y precipitación. $\mathrm{La}$ tasa de explotación ( $\mathrm{E}=0.61 /$ año en machos, $\mathrm{E}=0.60 /$ año en hembras) indica que el recurso se encuentra en una fase de sobrepesca donde la explotación pesquera aumenta la mortalidad; por consiguiente se sugiere cuidar que no se violen las épocas de veda, establecer un peso mínimo de captura de $1000 \mathrm{~g}$, revisar la tendencia de las capturas y proponer un $\mathrm{E}$ entre 0.4 y $0.5 /$ año con la finalidad de alcanzar el desarrollo sostenible y distribución equitativa de los recursos.

\section{AGRADECIMIENTOS}

Al Consejo de Investigación de la Universidad de Oriente por el co-financiamiento del
Proyecto de Grupo: "Evaluación de la pesquería artesanal de la comunidad de El Tirano, isla de Margarita" Código CI-6-030603-1587/2009. A Juan Miguel Rodríguez, Presidente de la Asociación de Pescadores Virgen del Carmen de El Tirano por su apoyo logístico. A Damerlín Cotúa Marcano por su colaboración en los muestreos de campo. A Miguel Cabrera del Centro de Investigación y de Estudios Avanzados del Instituto Politécnico Nacional-Unidad Mérida, México y a Clark Casler de la Universidad del Zulia, Venezuela por la lectura y sugerencias del manuscrito. A Atenas Guerrieri por su ayuda en el mejoramiento de las figuras. A los árbitros por sus acertados comentarios que permitieron mejorar el trabajo.

\section{RESUMEN}

En el estado Nueva Esparta, Venezuela, la pesca del pulpo Octopus vulgaris es considerada por los pescadores artesanales como una actividad alternativa para diversificar la producción y aumentar los ingresos, pero en los últimos años han venido experimentando fluctuaciones interanuales considerables. La necesidad de gestionar el recurso de una forma racional y responsable, impuso analizar algunos aspectos de la reproducción, crecimiento, mortalidad y pesquería, de modo que sirvan de base científica para llevar a cabo estrategias de manejo. Con el fin de cumplir con los objetivos, las muestras se obtuvieron durante la temporada de pesca junio-diciembre 2012 con una periodicidad semanal. A cada uno de los especímenes se le registró la longitud del manto $(\mathrm{Lm})$, peso total $(\mathrm{Pt})$, sexo y estado de madurez gonádica. Se procesaron 1268 machos de 9 a $25 \mathrm{~cm} \mathrm{Lm} \mathrm{y} 818$ hembras de 9 a $22 \mathrm{~cm}$ $\mathrm{Lm}$; la proporción de sexo mensual fue diferente a uno excepto junio y agosto, con un alto porcentaje de maduros en ambos sexos. Las tallas mínima y media de madurez sexual en machos fueron: $11 \mathrm{~cm}(428 \mathrm{~g})$ y $16 \mathrm{~cm}(1142 \mathrm{~g})$; mientras que en hembras $12 \mathrm{~cm}(476 \mathrm{~g})$ y $15.35 \mathrm{~cm}(844 \mathrm{~g})$. La relación longitud-peso de machos: $\mathrm{Pt}=0.7994 * \mathrm{Lm}^{2.62}$ y hembras: $\mathrm{Pt}=1.4552 * \mathrm{Lm}^{2.33}$ expresaron un crecimiento alométrico minorante. La estimación del crecimiento se basó en el análisis de la distribución de frecuencia de longitudes, para ello se usó el software FiSAT. Los parámetros de crecimiento estimados del modelo de von Bertalanffy fueron en machos: $\mathrm{L}_{\infty}=26.26 \mathrm{~cm}, \mathrm{P}_{\infty}=3769 \mathrm{~g}, \mathrm{k}=2.3 / \mathrm{año}$, $\mathrm{t}_{\mathrm{o}}=-0.015 / \mathrm{año} \mathrm{y}$ en hembras: $\mathrm{L}_{\infty}=24.28 \mathrm{~cm}, \mathrm{P}_{\infty}=2287 \mathrm{~g}$, $\mathrm{k}=1.8 / \mathrm{año}, \mathrm{t}_{\mathrm{o}}=-0.09 / \mathrm{año}$, mostraron un crecimiento rápido. La edad límite en machos fue de 1.30 años y en hembras de 1.57 años. Se observó asociación significativa y positiva de la captura del pulpo con la temperatura superficial del mar y negativa con la velocidad del viento y precipitación, que corresponde con la variabilidad hidrológica estacional 
de la zona de estudio. Se calculó la tasa de explotación actual ( $E=0.61 /$ año en machos; $E=0.60 /$ año en hembras) a través de los valores de mortalidad total $(Z=7.73$ /año en machos; $Z=6.63 /$ año en hembras), por pesca $(F=4.7 /$ año en machos; $F=3.99 /$ año en hembras) y natural $(M=3.03 /$

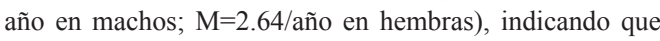
el pulpo se encuentra sobreexplotado; por consiguiente se recomienda regular la pesquería, reduciendo la tasa de mortalidad por pesca.

Palabras clave: Octopus vulgaris, reproducción, crecimiento, mortalidad, pesca, Venezuela.

\section{REFERENCIAS}

Bertalanffy, L. von. (1938). A quantitative theory of organic growth. Human Biology, 10(2), 181-213.

Boyle, P. R., \& Rodhouse, P. (2005). Cephalopods: Ecology and fisheries. Oxford, USA: Blackwell.

Cotúa, D. (2012). Determinación de la madurez sexual, relación talla-peso y factor de condición del pulpo común Octopus vulgaris Cuvier, 1797 del archipiélago Los Frailes, durante las temporadas de pesca 2010-2011 (Tesis Pregrado). Universidad de Oriente, Boca del Río, Venezuela.

Csirke, J. (1980). Introducción a la dinámica de poblaciones de peces (Documento Técnico de Pesca 192). Roma, Italia: FAO.

Demarcq, H., \& Faure, V. (2000). Coastal upwelling and associated retention indices derived from Satellite SST. Application to Octopus vulgaris recruitment. Acta Oceanologica, 23(4), 391-408.

Fernández-Núñez, M. M., Hernández-González, C. L., Raya, C. P., \& Balgueiúas, E. (1996). Reproductive biology of octopus Octopus vulgaris Cuvier, 1797 from North West African coast (21 "N - 26" $N)$. ICES Shellfish Committee Doc. C. M. 1996/K. (mimeo).

Froesch, D., \& Marthy, H. (1975).The structure and function of the oviducal gland in octopods (Cephalopoda). Biological Sciences Process, 188, 95-101.

Forsythe, J. W., \& van Heukelem, W. F. (1987). Growth. In P. Boyle (Ed.), Cephalopod Life Cycles. Vol. II. (pp. 135-156). London: Academic Press.

Gayanilo, F. C. Jr., Sparre, P., \& Pauly, D. (1996). FAOICLARM stock assessment tools (FiSAT). User's guide. FAO Computerized Information Series (Fisheries) 8 , Rome, FAO.

González, L. W., Euán, J., Eslava, N., \& Suniaga, J. (2007). La pesca de sardina, Sardinella aurita (Teleostei: Clupeidae) asociada a la variabilidad ambiental del ecosistema de surgencia costera de Nueva Esparta, Venezuela. Revista Biología Tropical, 55(1), 279-286.
González, L. W., Eslava, N., \& Guevara, F. (2001). La pesca artesanal del pulpo (Octopus spp.) en El Tirano, Isla de Margarita, Venezuela. Ciencia, 9(1), 18-27.

Guerra, A. (1975). Determinación de las diferentes fases del desarrollo sexual de Octopus vulgaris, mediante un índice de madurez. Investigación Pesquera, 39(2), 397-416.

Guerra, A. (1992). Mollusca Cephalopoda. Fauna Ibérica. Vol. 1. Museo Nacional de Ciencias Naturales. Consejo Superior de Investigaciones Científicas, Madrid, España.

Guerra, A., \& Manríquez, M. (1980). Parámetros biométricos de Octopus vulgaris. Investigación Pesquera, 44(1), 177-198.

Gulland, J. A. (1971). The fish resources of the oceans. Fishing News Books Ltd., Surrey, England.

Hernández-García, V., Hernández-López, J. L., \& CastroHernández, J. J. (2002). On the reproduction of Octopus vulgaris off the coast of the Canary Islands. Fisheries Research, 57(2), 197-203.

Hernández-López, J. L. (2000). Biología, ecología y pesca del pulpo común (Octopus vulgaris, Cuvier 1797) en aguas de Gran Canaria (Tesis Doctoral). Universidad de Las Palmas de Gran Canaria, España.

Hernández-Sánchez, A., \& De Jesús-Navarrete, A. (2010). Parámetros de crecimiento, mortalidad y tasa de explotación del pulpo Octopus maya en Holbox, Quintana Roo, México. Revista Biología Marina y Oceanografia, 45(3), 415-421.

Heukelem, W. F. van. (1979). Environmental control of reproduction and life span in Octopus: An Hypothesis. In S. E. Stancyk (Ed.), Reproductive ecology of marine invertebrates (pp. 123-133). Columbia: University of Carolina Press.

Hoydal, K., Rorvick, C., \& Sparre, P. (1982). Estimation of effective mesh sizes and their utilization in assessment. Dana, 2, 69-95.

Iglesias, J., Sánchez, F. J., \& Otero, J. J. (1997). Primeras experiencias sobre el cultivo integral del pulpo (Octopus vulgaris Cuvier) en el Instituto Español de Oceanografía. In J. Costa, E. Abellán, B. García, A. Ortega, \& S. Zamora (Eds.), Actas del VI Congreso Nacional de Acuicultura (pp. 221-226). Cartagena.

Jiménez-Badillo, M. L., del Río-Rodríguez, R. E., GómezSolano, M. I., Cu-Escamilla, A., \& Méndez-Aguilar, D. (2008). Madurez gonádica del pulpo Octopus vulgaris en el Golfo de México: análisis macroscópico y microscópico. Universidad Autónoma de Campeche, México.

Johnson, R., \& Wichern, D. (1992). Applied multivariate statistical analysis. $3^{\text {th }}$ ed. New Jersey, USA: Prentice-Hall Int. 
Katsanevakis, S., \& Verriopoulos, G. (2004). Abundance of Octopus vulgaris on soft sediment. Scientia Marina, 68(4), 553-560.

Mangold, K. (1983). Food, feeding and growth in cephalopods. Memoirs of the National Museum Victoria, 44, 81-93.

Mangold, K. (1987). Reproduction. In P. Boyle (Ed.), Cephalopod life cycles (Vol. II, pp. 150-200). London: Academic Press.

Mangold, K., \& Boletzky, S. V. (1973). New data on reproductive biology and growth of Octopus vulgaris. Marine Biology, 19, 7-12.

Muller-Karger, F., \& Aparicio, R. (1994). Mesoscale processes affecting phytoplankton abundance in the southern Caribbean Sea. Continental Shelf Research, 14(2-3), 199-221.

Okuda, T. (1974). Características oceanográficas generales de la costa suroriental del Mar Caribe. En Cuadernos Azules 15, Revisión de los datos oceanográficos en el Mar Caribe Suroriental especialmente en el margen continental de Venezuela (pp. 58-69). Universidad de Oriente, Cumaná, Venezuela.

Otero, J. (2006). Ecología del pulpo común (Octopus vulgaris Cuvier, 1797) en el área de afloramiento costero (Galicia, NE Atlántico) (Tesis Doctoral). Universidad de Vigo, España.

Otero, J., Álvarez-Salgado, X. A., González, A. F., Miranda, A., Groom, S. B., Cabanas, J. M., Casas, G., Wheatley, B., \& Guerra, A. (2008). Bottom-up control of common octopus Octopus vulgaris in the Galician upwelling system, northeast Atlantic Ocean. Marine Ecology Progress Series, 362, 181-192.

Pauly, D. (1979). Theory and management of tropical multispecies stocks: a review, with emphasis on the Southeast Asian demersal fisheries. International Center for Living Aquatic Resources Management, Studies and Review, 1, 1-35.

Pauly, D. (1980). On the interrelationships between natural mortality, growth parameters and mean environmental temperature in 175 fish stocks. International Council for the Exploration of the Sea, 39(2), 175-192.

Perales, C. (2001). Determinación de la edad y estudio del crecimiento del choco (Sepia hierredda Rang, 1837), el calamar (Loligo vulgaris Lamarck, 1798) y el pulpo (Octopus vulgaris Cuvier, 1797) de la costa noroccidental africana (Tesis Doctoral). Universidad de La Laguna, España.

Pereiro, J. A., \& Bravo de Laguna, J. (1979). Dinámica de la población y evaluación de los recursos del pulpo del Atlántico Centro-Oriental. Boletín del Instituto Español de Oceanografia, 5, 69-105.

Powell, D. (1979). Estimation of mortality and growth parameters from the length frequency of a catch. Rapport process-V Réunion Conseil International pour $L^{\prime}$ Exploitation de la Mer, 175, 167-169.
Quetglas, A., Alemany, F., Carbonell, A., Merella, P. \& Sánchez, P. (1998). Biology and fishery of Octopus vulgaris Cuvier, 1797 caught by trawlers in Mallorca (Balearic Sea, western Mediterranean). Fisheries Research, 36, 237-249.

Ricker, W. (1975). Computation and interpretation of biological statistic of fish population. Department of Environmental Fisheries and Marine Service. Bulletin of Fisheries Research Board of Canada, 191, 382.

Robaina, G. (1986). Guía práctica para el conocimiento de los pulpos de las costas de Venezuela. Contribuciones Cientificas, 10, 1-40.

Rocha, F. (2003). Biología, ecología, cultivo y pesquería de cefalópodos. Curso de Postgrado. Universidad Austral de Chile. Instituto de Investigaciones Marinas (CSIC) c/Eduardo Cabello 6, 36208 Vigo, España.

Rocha, F., Guerra, A., \& González, F. (2001). A review of reproductive strategies in cephalopods. Biological reviews of the Cambridge Philosophical Society, 76, 291-304.

Rodríguez-Rúa, A., Pozuelo, M., Prado, M., Gómez, M., \& Bruzón, M. A. (2005). The gametogenic cycle of Octopus vulgaris (Mollusca: Cephalopoda) as observed on the Atlantic coast of Andalusia (south of Spain). Marine Biology, 147, 927-933.

Roper, C. F. E., Sweeney, M. J., \& Huchberg, F. G. (1995). Cephalopodos. In W. Fisher, F. Krupp, W. Schneider, C. Somer, K. E. Carpenter, \& v. H. Niem (Eds.). Guía FAO para la identificación de especies para los fines de la pesca. Pacifico Centro Oriental (pp. 305-355). Roma: FAO.

Sokal, R., \& Rohlf, F. (1995). Biometry (3rd ed.). New York, USA: W.H. Freeman and Company.

Sparre, P., \& Venema, S. C. (1997). Introducción a la evaluación de recursos pesqueros tropicales. Parte 1. Manual (Documento Técnico de Pesca 306. 1 Rev. 2). Roma, Italia: FAO.

Steel, R., \& Torrie, J. (1985). Bioestadística: Principios y procedimientos. Bogotá, Colombia: Mc. Graw-Hill, Latinoamericana S.A.

Taylor, C. (1958). Cod growth and temperature. Journal of Conseil International pour L'Exploitation de la Mer, 23, 366-370.

Tresierra, A., \& Culquichicón, Z. (1993). Biología Pesquera. Trujillo, Perú: Editorial Libertad.

Warnke, K. (1999). Observations on the embryonic development of Octopus mimus (Mollusca: Cephalopoda) from northern Chile, Veliger, 42, 211-217.

Wetherall, J. (1986). A new method for estimating growth and mortality parameters from length-frequency data. Fishbyte, 4, 12-14.

Zar, J. H. (1996). Biostatistical analysis. Nueva Jersey, USA: Prentice Hall. 